\section{D) Check for updates}

Cite this: Food Funct., 2017, 8, 4547

\title{
The development of phytosterol-lecithin mixed micelles and organogels
}

\author{
Andrew B. Matheson, (D) *a Georgios Dalkas, (DD ${ }^{b}$ Andrei Gromov, ${ }^{c}$ \\ Stephen R Euston (D) ${ }^{b}$ and Paul S. Clegg (D) ${ }^{a}$
}

\begin{abstract}
We demonstrate that by mixing the phytosterol-ester oryzanol with lecithin in an organic solvent, both components may be dispersed at much higher concentrations than they may be individually. Dynamic light scattering and molecular dynamics simulations show that the mechanism for this is the formation of $r \sim 4 \mathrm{~nm}$ mixed micelles. Infrared spectroscopy and simulations suggest that these micelles are formed due in part to hydrogen bonding of the phosphate of the lecithin head-group, and the phenol group of the oryzanol. Rheology shows that by mixing these materials at an equimolar ratio, highly viscous suspensions are created. Furthermore, by adding water to these samples, a solid-like gel may be formed which offers mechanical properties close to those desired for a margarine type spread, whilst still solubilizing the oryzanol.
\end{abstract}

Received 16th August 2017, Accepted 28th October 2017 DOI: $10.1039 / \mathrm{c} 7 \mathrm{fo} 01271 \mathrm{c}$ rsc.li/food-function
These molecules are defined by a hydrophilic phosphatidylcholine headgroup and two hydrophobic fatty acid tails. Lecithin exhibits a rich and varied phase behavior, especially in ternary or quaternary systems. ${ }^{13,14}$ It may assemble into bilayers, lamellae, liposomes, or spherical or wormlike micelles depending on the solvent environment in which it is placed, and which other co-surfactants are present. Due to this, lecithin has been used to form organogels when blended with a wide range of materials. ${ }^{15-18}$ These molecules include a variety of steroids, which share similar structures to oryzanol. ${ }^{16,19,20}$ Particularly, bile salts have been shown to form wormlike micelles with lecithin which greatly increase the viscosity of a range of oils, and these gels have been shown to be compatible with water. ${ }^{14}$

With this in mind, we explored the behavior of systems containing both oryzanol and lecithin to investigate whether they form organogels in host oils with and without water, and if so, whether their properties are suitable for personal care or food applications. The experimental results are corroborated by atomistic computational studies in an attempt to gain further insight on the structure of lecithin : oryzanol organogels.

\section{Materials \& methods}

Soy bean lecithin with $95 \%$ purity was purchased from Avanti Polar Lipids. The average molecular weight of the lecithin was $775 \mathrm{~g} \mathrm{~mol}^{-1}$, with $63 \%$ of the faty acid tails having an $18: 2$ composition. Sunflower oil and octane were purchased from Sigma-Aldrich, oryzanol was supplied by Unilever. Lecithin and oryzanol were weighed out in vials, solvent was added and

\footnotetext{
School of Physics and Astronomy, University of Edinburgh, James Clerk Maxwell Building, Edinburgh EH9 3FD, UK. E-mail: Andrew.Matheson@ed.ac.uk; Tel: +44 (o) 1316505263

${ }^{b}$ Institute of Mechanical, Process and Energy Engineering, Heriot-Watt University, Edinburgh EH14 4AS, UK

${ }^{c}$ School of Chemistry, University of Edinburgh, Joseph Black Building, Edinburgh, EH9 3FD, UK
} 


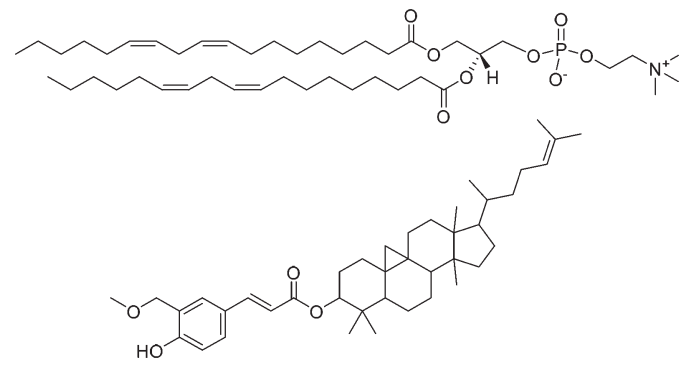

Fig. 1 Structure of lecithin (top) and oryzanol (bottom).

they were placed on a hotplate at $\sim 50{ }^{\circ} \mathrm{C}$ with magnetic stirrer bars. Raghavan et al. have established a technique for the preparation of lecithin organgogels in which the lecithin and co-gelator are dissolved in methanol, and then dried, to remove any water of crystallization. ${ }^{16}$ However, due to the poor solubility of oryzanol in methanol, we were unable to use this procedure. As such, there may be some water of crystallization still present in our systems. Once the materials were fully dissolved, the samples were taken from the hotplate to cool at room temperature. For gels with water added, lecithin-oryzanol solutions were weighed out and placed on a warm hotplate with a magnetic stirrer set at $\sim 1000 \mathrm{rpm}$ to disperse the water, the correct quantity of water was then added using an Eppendorf micropipette.

For rheology temperature sweeps, a TA Instruments DHR rheometer equipped with Peltier plate was employed. Crosshatched plate-plate geometry was used, and samples loaded in with spatula prior to measurement. An oscillatory stress of $1 \mathrm{~Pa}$ was applied at $1 \mathrm{~Hz}$, and samples allowed to equilibrate for $30 \mathrm{~min}$ at each temperature step before measurement. An AR2000 rheometer was used for all other rheology measurements. For systems prepared without water a $4 \mathrm{~cm} 1^{\circ}$ cone and plate geometry was chosen, and a cross-hatched plate geometry used for samples prepared with water, samples were loaded onto the bottom plate of the rheometer with a spatula. For steady state measurements a pre-shear of $10 \mathrm{~s}^{-1}$ was applied for $20 \mathrm{~s}$ before measurements, and adequate time left between measurements for samples to equilibrate. For oscillatory frequency sweeps, strain sweeps were carried out before hand to identify the linear regime. All rheology measurements were performed at room temperature, $\sim 20{ }^{\circ} \mathrm{C}$.

For the dynamic light scattering (DLS) measurements, samples were placed in test-tubes and sealed. Measurements were then performed using a $633 \mathrm{~nm}$ laser, with scattered light collected at $90^{\circ}$ from the incident beam direction, and then processed using an ALV-LSE-5004 correlator. Size distributions were obtained from mass weighted correlation functions.

For FTIR measurements, samples were dropcast onto IR reflective substrates. These were then imaged using a Renishaw inVia Raman Microscope with a non-contact all reflective objective, attached to a Smiths IlluminatIR FTIR spectrometer. Baseline spectra were subtracted from all sample spectra.
Confocal microscopy was performed using a Zeiss LSM T-PMT/LSM700 confocal laser scanning inverted microscope with Zeiss ZEN software (Carl Zeiss AG, Germany). The water phase was stained with Rhodamine-B dye to give contrast between aqueous and non-aqueous phases.

The 3D model of the sterol ester oryzanol was generated from SMILES representation using the program OMEGA $2.5^{21}$ and the conformation of lecithin was constructed using the CHARMM Ligand Reader and Modeler. ${ }^{22}$ Gasteiger charges were applied using AutoDockTools 1.5.6. ${ }^{23}$ The search space was defined by a grid box centered on lecithin with 80 grid points of $0.375 \AA$ spacing in each dimension. 100 docking rounds were calculated with AutoDock $4.2^{23}$ using the Lamarckian genetic algorithm with the default parameters of AutoDock4. The maximum number of energy evaluations was set to $5 \times 10^{6}$, and the solvent dielectric constant was set to 2.0, which is the dielectric constant of octane. The resulting docked conformations were clustered using a tolerance of $2.0 \AA$ and the most populated cluster with the highest free energy of binding $\Delta G$ was selected. Visual examination of the complexes and preparation of the figures were performed with AutodockTools and VMD 1.9. ${ }^{24}$

The initial configuration of the reverse mixed spherical micelle was constructed using the Packmol ${ }^{25}$ software package in order to satisfy all of the geometric constraints and to generate molecular packing with no overlaps. Taking into account the size distributions obtained for DLS (see results section), an aggregation number of 50 with $1: 1$ ratio of oryzanol to lecithin was selected for the formation of the inverse micelle of $\sim 3.5-4 \mathrm{~nm}$ radius. The total mass of each micelle was $35 \mathrm{kDa}$.

The AMBER v14 package ${ }^{26}$ with the latest Lipid14 ${ }^{27}$ force field, was used for simulation of the system. Force field parameters for the lipid were prepared according to the Lipid11 and Lipid14 force fields. ${ }^{27,28}$ Force-field parameters for oryzanol were prepared using the Antechamber package. ${ }^{29}$ Partial atomic charges were generated using the standard AMBER restraint electrostatic potential fit $(\mathrm{RESP})^{30}$ protocol from optimization of the initial conformation and calculation of the ESP at the HF/6-31G* level using Gaussian 09. ${ }^{31}$ The GAFF and Lipid14 force field parameters were applied to the structure using the XLEaP module of AMBER14.

A three-stage equilibration protocol, consisting of energy minimization and molecular dynamics (MD), was used to eliminate any unfavourable contacts which may have occurred as a result of model building process and to gently adjust the initial structure to the molecular mechanics force field prior to production of MD simulations. The energy of the initial structure was first minimized using 5000 steps of steepest descent minimization followed by 5000 steps of conjugate gradient minimization. Next, the system was slowly heated to the production temperature of $300 \mathrm{~K}$ in two stages. All atoms of the reverse spherical micelle were restrained during the heating to the initial coordinates in order to prevent large structural deviations using a harmonic restraint force constant of $10 \mathrm{kcal}$ $\mathrm{mol}^{-1} \AA^{-2}$. In the first heating stage the system target temperature was slowly heated from 0 to $100 \mathrm{~K}$ for $10 \mathrm{ps}$ with a con- 
stant volume simulation using the Langevin thermostat with a collision frequency of $1.0 \mathrm{ps}^{-1} .^{32}$ The second phase of heating slowly increased the temperature to the production temperature of $300 \mathrm{~K}$ over 90 ps with constant pressure periodic boundary conditions.

After heating the system, a production run of $50 \mathrm{~ns}$ of molecular dynamics was simulated at constant temperature and pressure using the Langevin thermostat at $300 \mathrm{~K}$ with a collision frequency of $1.0 \mathrm{ps}^{-1}$. The anisotropic Berendsen barostat had a reference pressure of 1.0 bar and a relaxation time of $2.0 \mathrm{ps}^{33}$ The SHAKE algorithm ${ }^{34}$ was used to constrain bonds involving hydrogen with a relative tolerance of $1 \times 10^{-7}$. The Particle Mesh Ewald (PME) method was used for longrange electrostatic and van der Waals interactions, ${ }^{35}$ with a real-space cut-off of $8 \AA$. A time step of 1 fs was used for equilibration and increased to 2 fs for the production of MD.

The first $20 \mathrm{~ns}$ of the simulation were considered as the initial relaxation period and were ignored during the data analysis. Analysis of the trajectories was performed using the CPPTRAJ module ${ }^{36}$ of AmberTools v16, and VMD was used to visualize the trajectories and prepare the figures. CPPTRAJ was used to find and track hydrogen bonds over the course of the trajectories by extracting their simple geometric features as a function of simulation time: the donor to acceptor heavy atom distance and the donor-hydrogen-acceptor angle. Both distance and angle cut-offs were equal to the default values for hydrogen bonds in CPPTRAJ.

\section{Results \& discussion}

Fig. 2 shows vials of $100 \mathrm{mM}$ lecithin in octane (left), $100 \mathrm{mM}$ lecithin and $100 \mathrm{mM}$ oryzanol in octane (centre), and $100 \mathrm{mM}$ oryzanol in octane (right). The lecithin sample is transparent, indicating that lecithin disperses readily in organic solvents at this concentration. This stands in contrast to the oryzanol sample, which is milky and opaque, due to the low solubility

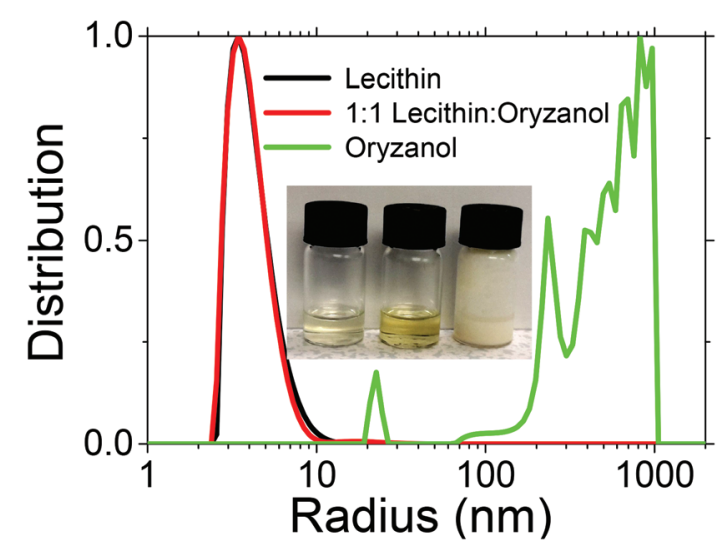

Fig. 2 Size distributions obtained from DLS of dispersions of $100 \mathrm{mM}$ lecithin in octane (black line, left photograph), $100 \mathrm{mM}$ lecithin and $100 \mathrm{mM}$ oryzanol in octane (red line, central photograph), and $100 \mathrm{mM}$ oryzanol in octane (green line, right photograph). of oryzanol. Looking at the mixed sample, we see that despite having the same concentration of oryzanol, it is transparent. This indicates that the addition of lecithin encourages the breakup of oryzanol aggregates and solubilizes the material. Looking at the size distributions obtained using DLS in Fig. 2, we see that data for the mixed system is almost identical to that which we obtain for a system without oryzanol, whereas the size of the scattering particles in the oryzanol only system is several orders of magnitude larger. This suggests that the mixed system results in the formation of inverse mixed micelles which are very similar in size to those formed by pure lecithin, i.e. inverse micelles of $\sim 4 \mathrm{~nm}$ radius.

FTIR may be used to better understand how the oryzanol is incorporated into these micelles, with results shown in Fig. 3. The majority of the features in the spectra of the blended system are consistent with a superposition of the spectra of the individual components. However, there are differences. In the oryzanol system we see a peak at $1265 \mathrm{~cm}^{-1}$, which has previously been assigned to the oryzanol $\mathrm{C}-\mathrm{O}$ ester bridge stretching, ${ }^{37}$ but that is absent in the blended system. A novel band appears at $1284 \mathrm{~cm}^{-1}$ which may be due to changes in the aforementioned $\mathrm{C}-\mathrm{O}$ stretching or to an asymmetric $\mathrm{O}-\mathrm{CH}_{3}$ deformation, as well as the significant growth of a band at $1127 \mathrm{~cm}^{-1}$, which may be assigned to methyl rocking. ${ }^{37}$ Most
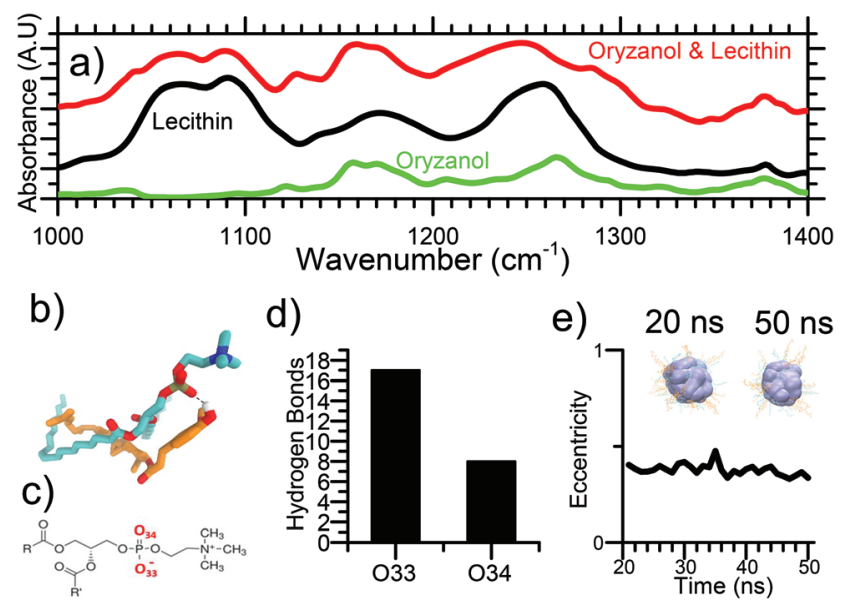

Fig. 3 (a) FTIR spectra of $100 \mathrm{mM}$ dispersions of oryzanol (green line), lecithin (black line) and mixed oryzanol and lecithin (red line), drop-cast on a reflective substrate (data sets offset for clarity). (b) Molecular docking simulation of lecithin (carbons are cyan, oxygen is red, nitrogen is blue, and phosphorus is khaki) and oryzanol (carbon is orange; oxygen is red; crucial hydrogen is white), showing the presence of an intermolecular hydrogen bond (dashed line). (c) The structure of the lecithin's lipid head group to clarify the labelling of oxygen atoms for (d) the number of hydrogen bonds of the oxygen atoms of lecithin's phosphate group (25 in total, one for each lecithin molecule) with the hydroxyl group of oryzanol. (e) Eccentricity of the reverse micelle over the last $30 \mathrm{~ns}$ of the simulation. Inset are simulation snapshots corresponding to a configuration of the mixed system at $20 \mathrm{~ns}$ of simulation time, and the final configuration of the mixed system after 50 ns. Lecithin's lipid head group and oryzanol's ferulic acid group are rendered as a mauve surface, lecithin's tails as cyan lines and oryzanol's ring system and alkyl chain as orange lines. 
significantly, for lecithin in octane we see a peak at $1258 \mathrm{~cm}^{-1}$ (which is consistent with an $\mathrm{OPO}^{-}$group where there is some water of crystallization present ${ }^{20,38}$ ) which shifts to $1247 \mathrm{~cm}^{-1}$ upon addition of oryzanol. There is not a peak in this region for oryzanol, but is consistent with the $\mathrm{OPO}^{-}$group undergoing intermolecular hydrogen bonding. ${ }^{20,38}$ Molecular docking simulations, see Fig. 3(b), are consistent with this, showing a hydrogen bond forming between the $\mathrm{OPO}^{-}$group of the lecithin and the $\mathrm{OH}$ group of the oryzanol.

Atomic level MD simulations have evolved as a complementary technique for the study of micelles. Some previous research has been focused on more complicated systems consisting of mixtures of lipids with molecules of biological interest, such as AOT. ${ }^{39,40}$

In service of a similar goal, we employed all-atom MD simulations to study the mixed system of lecithin-oryzanol. Fig. 3d presents the number of hydrogen bonding interactions between the lipid head groups and the oryzanol during the MD simulation time. The MD simulation data shows that the hydroxyl group of the oryzanol forms favourable hydrogen bonds with the oxygen atoms of the $\mathrm{OPO}^{-}$group of the lecithin. In addition, MD data analysis reveals that this hydrogen bond is very stable (for over $99 \%$ of the simulation time), and strongly indicates the proximity of the ferulic group of oryzanol to the lipid head group. To analyse the overall shape of the mixed reverse micelles, the moments of inertia, the semiaxes, and the eccentricity $(e)$ were calculated by assuming that the mixed reverse micelles are on average elliptical. The moments of inertia $I_{1}, I_{2}, I_{3}$ for an ellipse with semi-axes $a, b$ and $c$ are

$$
I_{1}=\frac{M}{5}\left(b^{2}+c^{2}\right), \quad I_{2}=\frac{M}{5}\left(c^{2}+a^{2}\right), \quad I_{3}=\frac{M}{5}\left(a^{2}+b^{2}\right)
$$

where $M$ is the mass of the mixed reverse micelles. The eccentricity was subsequently computed as

$$
e=\sqrt{1-\frac{c^{2}}{a^{2}}}
$$

For a sphere $I_{1} \approx I_{2} \approx I_{3}$ and $a \approx b \approx c$, and therefore the eccentricity is zero, and close to 1 for disc- and rod-like shapes. $^{39,40}$ The deviation from a spherical system can be expressed by examining the eccentricity in addition to simple visual inspection of snapshots of the mixed system. Fig. 3(e) shows the eccentricity of the mixed lecithin-oryzanol system as a function of simulation time for the final $30 \mathrm{~ns}$. It is clear that the mixed system has a stable spherical shape, with an average eccentricity of around 0.4 over the course of the simulation. On the other hand, a recent MD simulation study of lecithin reverse micelles showed an average eccentricity of around $0.6^{41}$ and a clear disc-to-rod transition during the simulation time.

It has previously been shown that for a polar molecule to cause a transition from spherical to wormlike micelles in a lecithin in oil system, the formation of hydrogen bonds with the phosphate head-group is not enough in itself. For instance, divalent inorganic salts may trigger the shift to wormlike micelles whist monovalent salts will not, ${ }^{17}$ ortho-coumaric acid will not form wormlike micelles with lecithin however paracoumaric acid does, ${ }^{19}$ and furthermore there is a hierarchy in the ability of various bile salts to gel lecithin sols. ${ }^{38}$ This is because the driving force for the transition from spherical to wormlike micelles is the change in the ratio of hydrophobic head group area to tail cross-sectional area, known as the packing parameter. From the docking and molecular dynamic simulations we see that the majority of the oryzanol molecule sits between the hydrophobic tails, and hence it does not penetrate as far as the choline moiety of the head-group. Therefore the packing parameter is not altered enough to change the size and shape of micelles, which remain spherical and not elongated.

The rheology of mixed lecithin-oryzanol systems is presented in Fig. 4a. The samples prepared with $R=$ [conc. oryzanol]/[conc. lecithin] $<0.7$ clearly exhibit shear thinning behavior. For samples prepared with $R>0.7$, at high shear rates the resulting stress exceeded the maximum value allowed by the rheometer before highly pronounced shear thinning could be observed, although there is still a clear downward trend in the
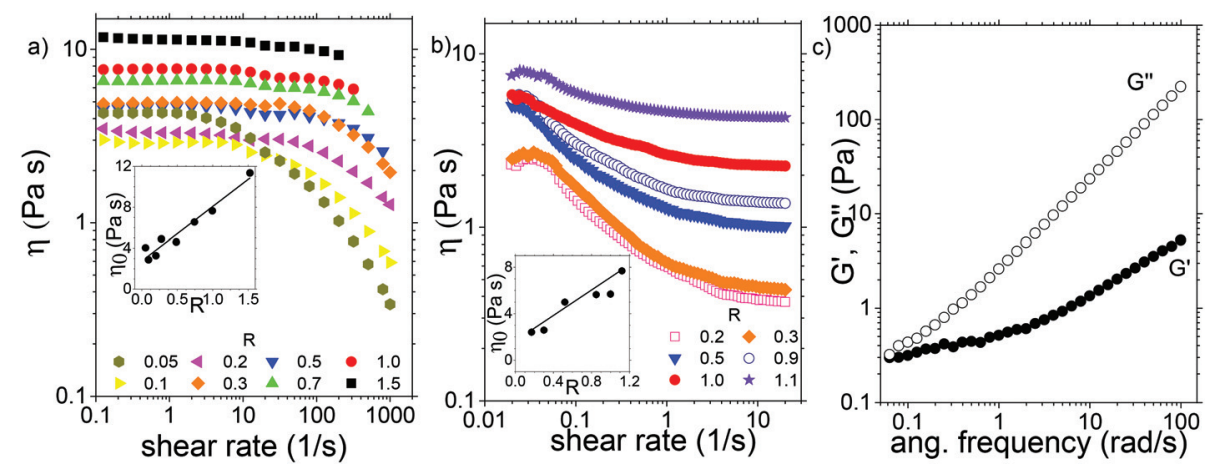

Fig. 4 Steady shear rheology measurements of samples prepared with $400 \mathrm{mM}$ lecithin (a) in octane and (b) sunflower oil with varying values of $R$, the molar ratio of oryzanol to lecithin concentration. Inset in each window is the zero shear viscosity extrapolated from low shear rate. (c) Oscillatory rheology of an $R=1$ sample in sunflower oil in the linear viscoelastic regime. All measurements were performed at $20^{\circ} \mathrm{C}$. 
viscosity with shear rate. Inset in Fig. 4(a) is a plot which shows that the zero shear viscosity increases linearly with $R$ up to a value of $R=1.5$, which is the maximum molar ratio we can achieve with the sample still transparent and homogenous. This stands in contrast to that observed in lecithin based wormlike micellar systems, in which there is typically a highly non-linear relationship between the molar ratio of materials and zero shear viscosity. ${ }^{16,17,19,42}$ This is consistent with the DLS and MD simulations which show that the addition of oryzanol does not result in a significant change in micelle size. Given that we believe the oryzanol does not change the size or shape of micelles, it raises the question of why the addition of oryzanol changes the viscosity of the system at all? There are two possible explanations - either the addition of oryzanol changes the local dielectric constant in a manner to increase the inter-micellar interactions, or the oryzanol effectively displaces some lecithin from each micelle, forcing additional micelles to form.

Initial results were obtained using octane, a simple alkane, as this solvent provides a "clean" background, free of residual lecithin which may be present in other plant derived oils, and give us a clear understanding of the structure of the inverse micelles. However, as we are interested in this system in the context of personal care and food-stuffs, it was important to explore how these materials behaved in a food safe solvent, such as sunflower oil.

Fig. 5(a) shows a selection of samples prepared with $400 \mathrm{mM}$ lecithin in sunflower oil, with varying values of $R$, the molar ratio of oryzanol to lecithin in solutions. Starting from the low $R$, left, we see the samples are very opaque and hazy due to the formation of lecithin aggregates. As

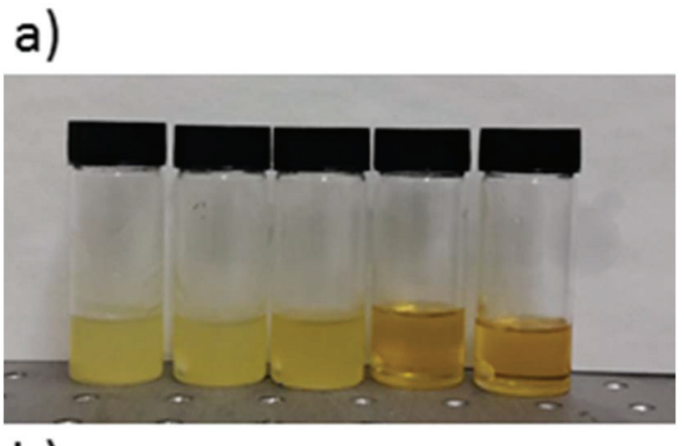

b)

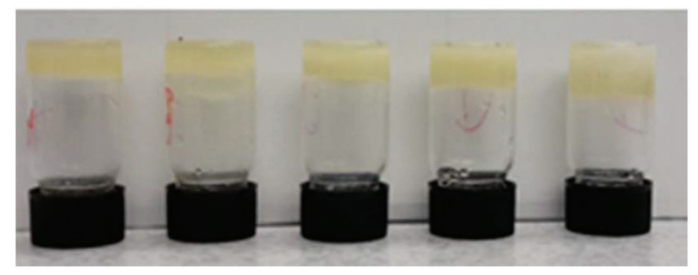

Fig. 5 (a) Vials of $\sim 400 \mathrm{mM}$ lecithin in sunflower oil, with (from left to right) $R=0,0.04,0.16,0.51,1.1$. (b) Gels prepared by mixing $400 \mathrm{mM}$ lecithin in sunflower oil $R=1 \mathrm{gel}$ with (from left) $4 \%, 9 \%, 18 \%, 30 \%, 43 \%$ water (by mass).
$R$ is increased (i.e. oryzanol is added to the system) the samples become increasingly transparent. This serves as a corollary to what we saw in Fig. 2, where adding lecithin to aggregated oryzanol dispersed the sterol. All the samples flow under inversion, but those on the left have significant solid like aggregates at the bottom of the vial. Upon the addition of lecithin the samples become homogenous and the solid-like lecithin phase at the base of the vial is dispersed, and once enough oryzanol has been added to disperse all the lecithin, further increases in $R$ result in an increase in viscosity. To quantify this, rheology was performed on homogenous samples, and is shown in Fig. 4(b). The data in Fig. 4(a) and (b) are similar; zero shear viscosity is in the 1-10 $\mathrm{Pa} \mathrm{s}$ range, and there is a linear relationship between zero shear viscosity and $R$. Additionally, samples prepared in both solvents exhibit shear-thinning behavior. There are, of course, differences between the samples prepared in octane and sunflower oil. The samples prepared in sunflower oil generally exhibit lower viscosities, and begin to shear thin at shear rates several orders of magnitude lower than the samples prepared in octane. This may be due to slight differences in solvent dielectric constant and viscosity altering how strongly micelles interact. However, the behavior is qualitatively similar enough to suggest that the lecithin and oryzanol form mixed micelles in a similar manner in both solvents. To further investigate the dynamics of the system, oscillatory rheology was performed on a sample with equimolar lecithin and oryzanol in sunflower oil, and is shown in the right hand panel of Fig. 4 . We can see that there is a frequency dependence in both $G^{\prime}$ and $G^{\prime \prime}$, but at high frequencies there is no cross-over into an elastic regime. Instead, we see that as frequency is increased, the system becomes less and less elastic. For wormlike micelles based on lecithin in organic solvents there is usually a cross-over from viscous to elastic behavior in the range of $\omega=$ $0.01-10,{ }^{16,19,38,42,43}$ although depending on the system this may occur at even lower frequencies. ${ }^{17}$ This gives further evidence that although adding oryzanol to a lecithin solution does result in an increase in viscosity, the mechanism for this is not the formation of wormlike micelles.

Having shown that lecithin may be used to solubilize oryzanol, and that the addition of oryzanol to lecithin in oil solutions may increase viscosity, it is worth considering the possible applications for this system in some more detail. Some research has been carried out on incorporating oryzanol into cosmetics due to its antioxidant activity, ${ }^{44}$ and topically applied oryzanol has been shown to inhibit the spread of skin tumors in mice. ${ }^{45}$ This inverse mixed micelle system may offer a means of increasing the amount of oryzanol delivered to the skin. As stated earlier, another major application for phytosterol organogels is in foodstuffs, particularly low-fat spreads, ${ }^{2,3}$ but the incompatibility of sitosterol-oryzanol organogels with water has proved a barrier to this. For that reason, we wanted to see how the phytosterol-lecithin mixed micellar system behaved in the presence of water.

Upon adding water to a sunflower oil sample with equimolar lecithin and oryzanol, it becomes more solid-like, result- 
ing in a gel which does not flow under inversion, as shown in Fig. 5(b). The samples are no longer transparent, which clearly indicates that there is scattering from microscopic objects in this system, rather than just nanoscale inverse micelles. Dilution tests show that these emulsions are water in oil in structure. At the highest concentration shown here (i.e. water content $>40 \%$ ), there is some free water, indicating a degree of phase separation or water not fully incorporated into the gel. Samples were also prepared using micellar systems with a lower value of $R=0.25$, but resulted in highly inhomegenous samples, which flowed upon inversion.

By observing that these $R=1$ samples do not flow readily after inversion, we know that the structural changes due to the addition of water have changed the rheological behavior. To quantify these changes we performed oscillatory rheology, with a stress sweep shown in Fig. 6a and a frequency sweep in Fig. 6b. We focus on an $R=1$ sample with $\sim 17 \%$ water content by mass, as this is in the range typical for butter, ${ }^{46,47}$ rather than offer an exhaustive exploration of the phase space. From stress sweeps we see solid-like behavior at low applied stresses followed by yielding, which is consistent with an emulsion with a high volume fraction of internal phase. ${ }^{48}$ The observed $G^{\prime}$ values and yield stress (the stress at which $G^{\prime \prime}>G^{\prime}$ ) of this system are far lower than that of sitosterol-oryzanol organogels prepared with a similar mass of sterol, ${ }^{4}$ and unlike sitosteroloryzanol gels it gently shear-thins rather than fracturing. Frequency sweeps performed in the linear viscoelastic regime show that $G^{\prime}>G^{\prime \prime}$ over the full frequency domain probed. Rheological properties such as this are favourable for spreadable fats, ${ }^{49}$ which suggests that this system could have possible applications as the basis of a low fat spread with cholesterol lowering properties. Additionally, the observed storage modulus is lower than that of butter, but is of the same order of magnitude. ${ }^{47,50}$ It is worth noting that these measurements were performed at $\sim 20{ }^{\circ} \mathrm{C}$ to maintain consistency with the measurements performed on anhydrous samples shown in Fig. 4. A temperature sweep was also performed on a sample of this composition, and shown in Fig. 6(c). It shows that although the gel softens at $T>35^{\circ} \mathrm{C}$, it remains its solid consistency over a broad temperature range.

We may gain a better understanding of the microstructure of the quaternary gels by examining them using fluorescence confocal microscopy. In Fig. $6 \mathrm{~b}$ we present a confocal image obtained for the $\sim 17 \%$ water sample, where the water phase has been dyed red using Rhodamine B. There are no clear signs of individual water droplets, although the deep red regions of the image show that there are obviously water rich regions of the gel. This would suggest that water is sequestered in small droplets/swollen micelles which are too small to observe directly but that tend to stick together. Recent work by Cautela et al. investigated a similar quaternary system of bile salt-lecithin-water-hexane, and observed that wormlike micelles still form as they would in either bile salt-lecithinhydrocarbon or water-lecithin-hydrocarbon ternary systems, but that micelles need not be exclusively lecithin-water or lecithin-bile in composition. ${ }^{14}$ It is not clear to us whether all the lecithin remains tightly bound to oryzanol and water is incorporated into the centre of micelles which swell, or whether some lecithin is sequestered away from mixed micelles to stabilize the droplets. Regardless, the gels have a

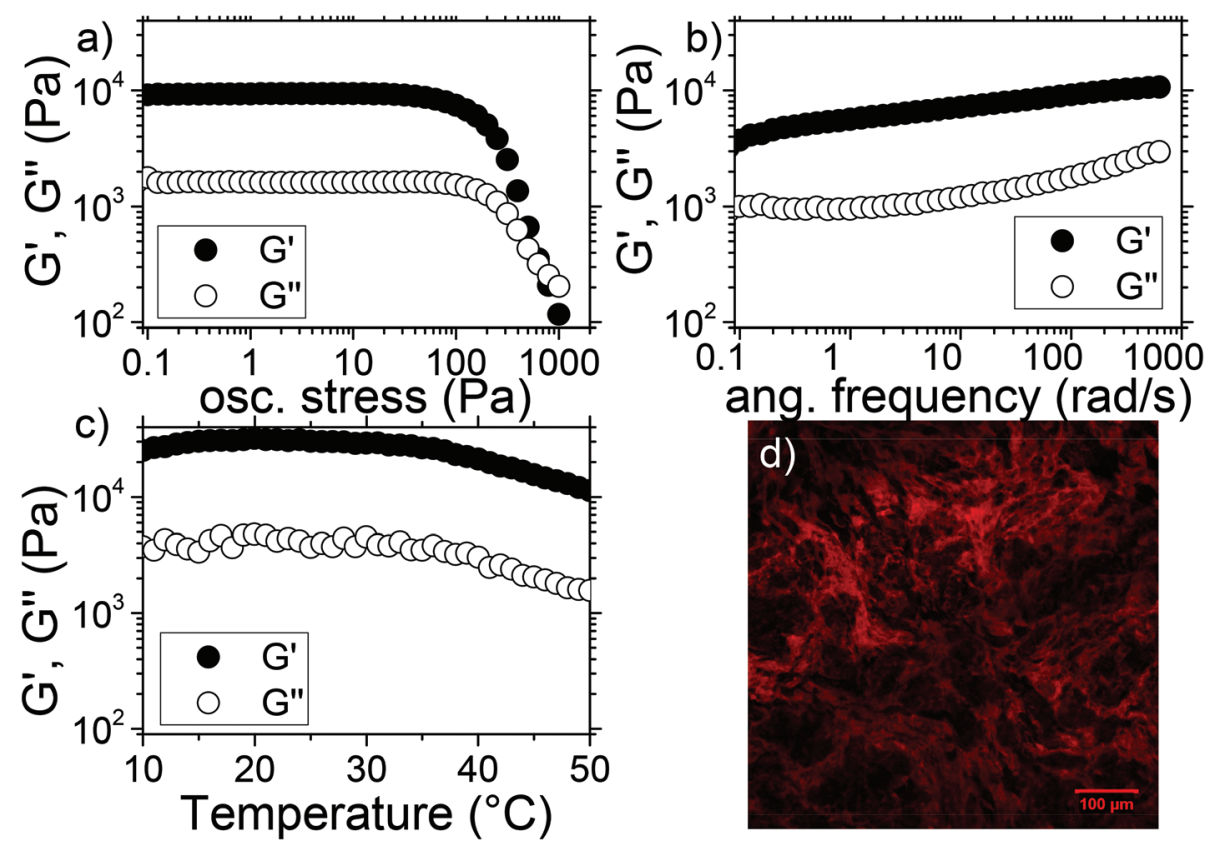

Fig. 6 (a) Oscillatory rheology stress sweep, (b) frequency sweep and (c) temperature sweep performed applying a 1 Pa oscillatory stress at $1 \mathrm{~Hz}$ on a sample prepared by blending $17 \%$ water into a suspension of $400 \mathrm{mM}$ lecithin $400 \mathrm{mM}$ oryzanol in sunflower oil, (d) confocal image of same gel, with water dyed red using Rhodamine $\mathrm{B}$. 
smooth, homogenous consistency with no sign of oryzanol precipitation, which suggests that there is adequate lecithin in the system to both stabilize droplets and solubilize oryzanol. This stands in contrast to sitosterol-oryzanol gels in the presence of water, where sitosterol-hydrate formation is thermodynamically favourable. $5,12,51$ This tendency for droplets to aggregate in lecithin stabilized water in oil emulsions to flocculate has been reported before, ${ }^{52,53}$ and we assume that van der Waals interactions between water droplets are the dominant mechanism. The observed structure may give us some insight into why a high value of $R$ is required to form homogenous gels (i.e. $R=1$ forms a gel whereas $R=0.25$ did not). The network appears to be formed of swollen micelles stabilized either by lecithin on its own or by lecithin and oryzanol, we proposed earlier that a system with equimolar oryzanol and lecithin may result in the formation of more inverse micelles than a system with only lecithin. It is thus plausible that if there is a higher number of micelles afforded by $R=1$, this allows- (a) an increase in the viscosity of anhydrous samples, (b) more water to be dispersed without phase separation, and (c) a denser network of swollen micelles to form the gel network. Alternatively, we also suggested that the addition of oryzanol may result in stronger inter-micellar interactions, and it may be that this is the key to why a high value of $R$ is required for gelation to occur.

\section{Conclusions}

In this work we have investigated the formation of highly viscous dispersions and organogels using a mixture of lecithin and the phytosterol oryzanol, both experimentally and computationally. The formation of these organogels proceeds through the formation of mixed micelles, mediated by intramolecular hydrogen bonding, and offer a means of vastly increasing the solubility of oryzanol in sunflower oil, and simplifying its incorporation into food stuff or personal care products. Moreover, the addition of oryzanol to lecithin solutions results in an increase in viscosity. The incorporation of water into the mixed micelle solution results in a change in the behavior, as it goes from being a highly viscous fluid to a more solid-like gel. The rheological properties of this system suggest that it may be suitable as the basis of a low-fat spread.

\section{Conflicts of interest}

The authors declare no competing interests.

\section{Acknowledgements}

We thank Arjen Bot of Unilever R\&D for useful conversations and materials. This work was supported by the BBSRC DRINC project. [Grant BBSRC BB/M027597/1 and BB/M027449/1]. This project made use of time on ARCHER granted via the UK High-End Computing Consortium for Biomolecular
Simulation, HECBioSim (http://hecbiosim.ac.uk), supported by EPSRC (grant no. EP/L000253/1).

\section{References}

1 M. B. Katan, S. M. Grundy, P. Jones, M. Law, T. Miettinen and R. Paoletti, Mayo Clin. Proc., 2003, 78, 965-978.

2 L. A. Simons, Am. J. Cardiol., 2002, 90, 737-740.

3 N. de Jong, A. Zuur, M. C. J. Wolfs, G. C. W. Wendel-Vos, J. M. A. van Raaij and A. J. Schuit, Eur. J. Clin. Nutr., 2007, 61, 1407-1415.

4 A. Bot and W. G. M. Agterof, J. Am. Oil Chem. Soc., 2006, 83, 513-521.

5 H. Sawalha, R. den Adel, P. Venema, A. Bot, E. Flöter and E. van der Linden, J. Agric. Food Chem., 2012, 60, 3462-3470.

6 A. Bot, E. P. Gilbert, W. G. Bouwman, H. Sawalha, R. den Adel, V. M. Garamus, P. Venema, E. van der Linden and E. Floter, Faraday Discuss., 2012, 158, 223-238.

7 H. Ritter, R. L. K. M. van de Sande and V. K. Müller, WO, 97/42830, 1997.

8 A. B. Matheson, V. Koutsos, G. Dalkas, S. Euston and P. Clegg, Langmuir, 2017, 33, 4537-4542.

9 C. S. O’Bryan, T. Bhattacharjee, S. Hart, C. P. Kabb, K. D. Schulze, I. Chilakala, B. S. Sumerlin, W. G. Sawyer and T. E. Angelini, Sci. Adv., 2017, 3, e1602800.

10 S. Basak, J. Nanda and A. Banerjee, J. Mater. Chem., 2012, 22, 11658-11664.

11 M. A. Rogers, A. J. Wright and A. G. Marangoni, Soft Matter, 2009, 5, 1594-1596.

12 R. den Adel, C. M. H. Patricia and B. Arjen, J. Phys.: Conf. Ser., 2010, 247, 012025.

13 A. Kabalnov, T. Tarara, R. Arlauskas and J. Weers, J. Colloid Interface Sci., 1996, 184, 227-235.

14 J. Cautela, M. Giustini, N. V. Pavel, G. Palazzo and L. Galantini, Colloids Surf., A, 2017, 352, 411-419.

15 M. Pernetti, K. F. van Malssen, E. Flöter and A. Bot, Curr. Opin. Colloid Interface Sci., 2007, 12, 221-231.

16 S.-H. Tung, Y.-E. Huang and S. R. Raghavan, J. Am. Chem. Soc., 2006, 128, 5751-5756.

17 H.-Y. Lee, K. K. Diehn, S. W. Ko, S.-H. Tung and S. R. Raghavan, Langmuir, 2010, 26, 13831-13838.

18 L. Han, L. Li, B. Li, L. Zhao, G.-Q. Liu, X. Liu and X. Wang, J. Am. Oil Chem. Soc., 2014, 91, 1783-1792.

19 R. Kumar, A. M. Ketner and S. R. Raghavan, Langmuir, 2010, 26, 5405-5411.

20 Z. Shervani, T. K. Jain and A. Maitra, Colloid Polym. Sci., 1991, 269, 720-726.

21 P. C. Hawkins, A. G. Skillman, G. L. Warren, B. A. Ellingson and M. T. Stahl, J. Chem. Inf. Model., 2010, 50, 572-584.

22 S. Jo, T. Kim, V. G. Iyer and W. Im, J. Comput. Chem., 2008, 29, 1859-1865.

23 G. M. Morris, R. Huey, W. Lindstrom, M. F. Sanner, R. K. Belew, D. S. Goodsell and A. J. Olson, J. Comput. Chem., 2009, 30, 2785-2791.

24 W. Humphrey, A. Dalke and K. Schulten, J. Mol. Graphics, 1996, 14, 33-38. 
25 L. Martínez, R. Andrade, E. G. Birgin and J. M. Martínez, J. Comput. Chem., 2009, 30, 2157-2164.

26 D. A. Case, V. Babin, J. T. Berryman, R. M. Betz, Q. Cai, D. S. Cerutti, T. E. Cheatham, T. A. Darden, R. E. Duke, H. Gohlke, A. W. Goetz, S. Gusarov, N. Homeyer, P. Janowski, J. Kaus, I. Kolossváry, A. Kovalenko, T. S. Lee, S. LeGrand, T. Luchko, R. Luo, B. Madej, K. M. Merz, F. Paesani, D. R. Roe, A. Roitberg, C. Sagui, R. SalomonFerrer, G. Seabra, C. L. Simmerling, W. Smith, J. Swails, R. C. Walker, J. Wang, R. M. Wolf, X. Wu and P. A. Kollman, \{Amber 14\}, 2014.

27 C. J. Dickson, B. D. Madej, Å. A. Skjevik, R. M. Betz, K. Teigen, I. R. Gould and R. C. Walker, J. Chem. Theory Comput., 2014, 10, 865-879.

28 Å. A. Skjevik, B. D. Madej, R. C. Walker and K. T. Eigen, J. Phys. Chem. B, 2012, 116, 11124-11136.

29 J. Wang, R. M. Wolf, J. W. Caldwell, P. A. Kollman and D. A. Case, J. Comput. Chem., 2004, 25, 1157-1174.

30 C. I. Bayly, P. Cieplak, W. Cornell and P. A. Kollman, J. Phys. Chem., 1993, 97, 10269-10280.

31 M. J. Frisch, G. W. Trucks, H. B. Schlegel, G. E. Scuseria, M. A. Robb, J. R. Cheeseman, G. Scalmani, V. Barone, B. Mennucci, G. A. Petersson, H. Nakatsuji, M. Caricato, X. Li, H. P. Hratchian, A. F. Izmaylov, J. Bloino, G. Zheng, J. L. Sonnenberg, M. Hada, M. Ehara, K. Toyota, R. Fukuda, J. Hasegawa, M. Ishida, T. Nakajima, Y. Honda, O. Kitao, H. Nakai, T. Vreven, J. A. Montgomery, J. E. Peralta, F. Ogliaro, M. Bearpark, J. J. Heyd, E. Brothers, K. N. Kudin, V. N. Staroverov, R. Kobayashi, J. Normand, K. Raghavachari, A. Rendell, J. C. Burant, S. S. Iyengar, J. Tomasi, M. Cossi, N. Rega, J. M. Millam, M. Klene, J. E. Knox, J. B. Cross, V. Bakken, C. Adamo, J. Jaramillo, R. Gomperts, R. E. Stratmann, O. Yazyev, A. J. Austin, R. Cammi, C. Pomelli, J. W. Ochterski, R. L. Martin, K. Morokuma, V. G. Zakrzewski, G. A. Voth, P. Salvador, J. J. Dannenberg, S. Dapprich, A. D. Daniels, Ö Farkas, J. B. Foresman, J. V. Ortiz, J. Cioslowski and D. J. Fox, Wallingford CT, 2009, DOI: citeulike-article-id:9096580.

32 R. W. Pastor, B. R. Brooks and A. Szabo, Mol. Phys., 1988, 65, 1409-1419.

33 H. J. C. Berendsen, J. P. M. Postma, W. F. V. Gunsteren, A. DiNola and J. R. Haak, J. Chem. Phys., 1984, 81, 3684-3690.
34 J.-P. Ryckaert, G. Ciccotti and H. J. C. Berendsen, J. Comput. Phys., 1977, 23, 327-341.

35 T. Darden, D. York and L. Pedersen, J. Chem. Phys., 1993, 98, 10089-10092.

36 D. R. Roe and T. E. Cheatham, J. Chem. Theory Comput., 2013, 9, 3084-3095.

37 E. Mandak, D. Zhu, T. A. Godany and L. Nyström, J. Agric. Food Chem., 2013, 61, 2446-2452.

38 C.-W. Njauw, C.-Y. Cheng, V. A. Ivanov, A. R. Khokhlov and S.-H. Tung, Langmuir, 2013, 29, 3879-3888.

39 S. Abel, F. Sterpone, S. Bandyopadhyay and M. Marchi, J. Phys. Chem. B, 2004, 108, 19458-19466.

40 A. V. Martinez, L. Dominguez, E. Małolepsza, A. Moser, Z. Ziegler and J. E. Straub, J. Phys. Chem. B, 2013, 117, 7345-7351.

41 S. Vierros and M. Sammalkorpi, J. Chem. Phys., 2015, 142, 094902.

42 P. Schurtenberger, R. Scartazzini, L. J. Magid, M. E. Leser and P. L. Luisi, J. Phys. Chem., 1990, 94, 36953701.

43 M. Imai, K. Hashizaki, H. Taguchi, Y. Saito and S. Motohashi, J. Colloid Interface Sci., 2013, 403, 77-83.

44 A. Viriyaroj, T. Ngawhirunpat, M. Sukma, P. Akkaramongkolporn, U. Ruktanonchai and P. Opanasopit, Pharm. Dev. Technol., 2009, 14, 665-671.

45 K. Yasukawa, T. Akihisa, Y. Kimura, T. Tamura and M. Takido, Biol. Pharm. Bull., 1998, 21, 1072-1076.

46 M. Kalab, J. Dairy Sci., 1985, 68, 3234-3248.

47 S. Rønholt, J. J. K. Kirkensgaard, T. B. Pedersen, K. Mortensen and J. C. Knudsen, Food Chem., 2012, 135, 1730-1739.

48 R. Pal, Food Hydrocolloids, 2006, 20, 997-1005.

49 A. J. Wright, M. G. Scanlon, R. W. Hartel and A. G. Marangoni, J. Food Sci., 2001, 66, 1056-1071.

50 A. Shukla and S. S. H. Rizvi, J. Food Sci., 1995, 60, 902-905.

51 T. Moschakis, E. Panagiotopoulou and E. Katsanidis, LWTFood Sci. Technol., 2016, 73, 153-161.

52 A. Knoth, I. Scherze and G. Muschiolik, Food Hydrocolloids, 2005, 19, 635-640.

53 I. Scherze, A. Knoth and G. Muschiolik, J. Dispersion Sci. Technol., 2006, 27, 427-434. 